Research Paper

\title{
Low Preoperative albumin-to-globulin ratio Predict Poor Survival and Negatively Correlated with Fibrinogen in Resectable Esophageal Squamous Cell Carcinoma
}

\author{
Xiao-Hui Li1 ${ }^{1 \dagger}$, Wen-Shen Gu²†, Xue-Ping Wang ${ }^{1}$, Jian-Hua Lin ${ }^{1}$, Xin Zheng ${ }^{1}$, Lin Zhang1,3, Ting Kang1, \\ Zhi-Xian Zhang ${ }^{2 \bowtie}$ and Wan-li Liu ${ }^{\boxplus}$ \\ 1. State Key Laboratory of Oncology in South China, Collaborative Innovation Center for Cancer Medicine, Department of Clinical Laboratory Medicine, Sun \\ Yat-Sen University Cancer Center, Guangzhou 510060, China \\ 2. Guangdong Provincial Key Laboratory of Malignant Tumor Epigenetics and Gene Regulation, Department of Clinical Laboratory Medicine, Sun Yat-Sen \\ Memorial Hospital, Sun Yat-Sen University, Guangzhou 510020, China \\ 3. Guangdong Esophageal Cancer Research Institute, Guangzhou 510060, China \\ † These authors contributed equally to this work. \\ $\square$ Corresponding authors: liuwl@sysucc.org.cn (W.-L.L.); zhixianzhang_11@163.com(Z.-X.Z). Tel./Fax: +86-20-8734-3196 ((W.-L.L.); +86-20-8133-2072 (Z.-X.Z) \\ ( $)$ Ivyspring International Publisher. This is an open access article distributed under the terms of the Creative Commons Attribution (CC BY-NC) license \\ (https://creativecommons.org/licenses/by-nc/4.0/). See http://ivyspring.com/terms for full terms and conditions.
}

Received: 2017.01.05; Accepted: 2017.04.26; Published: 2017.07.02

\begin{abstract}
Background: Although various inflammation-based indexes in esophageal carcinoma have been documented, but the prognostic value of the albumin-to-globulin ratio(AGR) and its correlation with fibrinogen in resectable ESCC remain unknown.

Methods: The levels of pre-treatment serum common acute phase proteins (including CRP, albumin and fribrinogen) were retrospectively analyzed in 447 patients with ESCC who underwent surgical resection at our department. The prognostic value was explored by univariate and multivariate cox hazard analysis. The correlation between AGR and acute phase proteins were also analyzed.

Results: Patients with decreased levels of AGR and increased CRP had significantly lower 5-year survival rates than those with higher AGR, not only in the whole ESCC cohort but also in the subgroups stratified according to the disease T, N classifications, and metastasis, whereas the other acute phase proteins were not independent prognostic factors for ESCC. In addition, a lower AGR level was observed more often in patients with a high fibrinogen level than in those with a low fibrinogen level. Spearman's rank correlation analysis revealed that the AGR level presented a negative correlation with the fibrinogen level $(r=-0.317, p<0.001)$.

Conclusions: The 5-year survival was shorter in resectable ESCC patients exhibiting decreased pre-treatment AGR and increased CRP. Thus, the serum AGR and CRP may be a clinical prognostic factor for resectable ESCC patients. In addition, a negative correlation was present between the levels of AGR and fibrinogen, the common indexes of acute phase reactants.
\end{abstract}

Key words: esophageal squamous cell carcinoma, albumin-to-globulin ratio, CRP, survival, fibrinogen

\section{Introduction}

Esophageal squamous cell carcinoma (ESCC) is the major pathological type of esophageal cancer and one of the most common digestive system malignancies worldwide, especially in some Asian countries, with China include[1, 2]. With the advanced surgical techniques and therapy, the 
outcome of ESCC have improved than before but the 5-year survival rate still poor[2, 3]. Recently increasing researches focus on the identification of various inflammation-based indexes in esophageal cancer, as well as various other cancers[4-6], such as the modified Glasgow Prognostic Score (mGPS)[7], the prognostic nutritional index (PNI)[8], the neutrophil-lymphocyte ratio(NLR) and the platelet-lymphocyte ratio (PLR)[7] and C-reactive protein-albumin(CRP/Alb) ratio[9-11].

Acute phase proteins are available inflammation markers applied in clinic widely which include CRP, albumin, fibrinogen, alpha1-acid glycoprotein, alpha1-antichymotrypsin, alpha1-antitrypsin, alpha2macroglobulin, ceruloplasmin, haptoglobin, and transferrin. Researchers found that the serum concentration of some acute phase proteins may correlate with the more aggressive clinical behavior of lung cancer[4, 10-12]. Hypoalbuminemia has recently been demonstrated to be a predictive factor of poor responsiveness[13, 14]. Besides, albumin-to-globulin ratio(AGR) is one of the most common tests used in the laboratory and its prognostic value in cancer has been documented in several cancer[15-17]. However, few studies have systematically investigated the relationship between ESCC and AGR, and the prognostic value of the AGR consequently remains unclear.

The aim of the present study was to assess the clinical and prognostic significance of AGR in Chinese patients with resectable ESCC. In addition, we analyzed the correlation of AGR with the common acute phase proteins in 447 patients with resectable ESCC.

\section{Methods}

\section{Inclusion criteria of patients}

This study examined 447 consecutive patients who received histologically confirmed diagnoses of ESCC between May 2007 and December 2009 at the Sun Yat-sen University Cancer Center in Guangzhou, China. Patients with one of the following conditions were excluded: (1) patients younger than 18 years of age or older than 80; (2) patients who received antitumor treatment or surgery before enrolling in this study, unable to surgery or not accept the operation; (3) patients with rheumatoid diseases and acute infection; (4) patients had clinical evidence of inflammation in nearly one month, immunity disease, hematology disease or end-stage liver disease. Using the patients' medical records, the grade of tumor differentiation and stage were classified based on the 7th edition of the AJCC/UICC TNM system[18]. In our cohort, all 447 patients underwent surgery of radical esophagectomy which included primary tumor resection and lymph node dissection. The most commonly used surgical approaches included the left transthoracic procedure, the Ivor-Lewis approach, and the cervical-thoracoabdominal procedure. Detailed clinical and pathological information, including demographic data, smoking status, pathological tumor, node, metastasis stage and overall survival data, were available for all patients. All the data can be found in the Research Data Deposit public platform (www.researchdata.org.cn) provided by RDD Management Committee in the Sun Yat-sen University Cancer Center and the RDD number for this dataset is RDDA2017000179.

\section{Ethics statement and follow-up of patients}

All 447 patients have provided written informed consent for their information to be stored and used in the hospital database. Study approval was obtained from independent ethics committees at Sun Yat-Sen University Cancer Center. Patients were followed up every three months for the first two years, then every six months for another three years and every one year for the rest of time. We consulted the out-patient information and made telephone interviews by a special follow-up department in our hospital to all patients to know their survival situation. The overall patient survival, defined as the time from surgery to death or last follow up, whichever came first, was used as a measure of prognosis.

\section{Laboratory Measurements}

Venous blood samples were collected in tubes prior to the initiation of any treatment.

$\mathrm{CRP}$ and Alb were measured using a Hitachi 7600-020 automatic biochemical analyzer(HITACHI, Tokyo, Japan). Serum albumin was measured using the bromocresol green(BCG) dye method, and serum CRP levels were assessed using latex agglutination turbidimetric immunoassays. Glb was calculated based on the TP and Alb: TP - Alb. AGR was defined as the absolute albumin value divided by the absolute globulin value. The level of fibrinogen measured by a Sysmex CA-7000 automatic coagulation analyzer (Sysmex Corporation, Kobe, Japan). Based on thrombin clotting times, fibrinogen was assayed according to the Clauss method. Data made available to all interested researchers upon request.

\section{Statistical Analysis}

We used $4.0 \mathrm{~g} / \mathrm{L}$ and $10.0 \mathrm{mg} / \mathrm{L}$ as cut-off point of fibrinogen and CRP respectively basing on their clinical significance. The median pretreatment AGR was 1.61 and we set 1.66 as the cut-off value based on receiver operating characteristics(ROC) curve with the largest Youden Index. All patients were classified 
into two groups according to the AGR. The correlation between AGR and clinical characteristics was assessed using the Mann-Whitney $U$ test or Kruskal-Wallis $\mathrm{H}$ and $X^{2}$ tests. Univariate and multivariate analyses of clinical variables were performed using Cox proportional hazards regression models. Variables significantly prognostic in univariate analysis were selected for multivariable analysis using the forward stepwise method to identify whether these independent variables could be used as an independent prognostic factor for survival. The results of this survey were analyzed using the Kaplan-Meier survival curves with the log-rank test. The association between AGR and fibrinogen was analyzed by Spearman's rank correlation. A nomogram for possible prognostic factors associated with OS was established by $R$ software with the Harrell's concordance index(c-index). $P$ values $<0.05$ were considered to indicate significant differences. All reported $P$ values are two sided, and all statistical tests were performed with the IBM SPSS Statistics version 22 for Windows (IBM Corporation, Armonk, NY, USA).

Table 1. Main Clinical Characteristics and Parameter in 447 Patients with ESCC

\begin{tabular}{|c|c|}
\hline Characteristics & $\begin{array}{l}\text { Median (25th-75th percentile) or } \\
\text { no.(\%) }\end{array}$ \\
\hline \multicolumn{2}{|l|}{ Gender(n) } \\
\hline Male & $346(77.4)$ \\
\hline Female & 101(22.6) \\
\hline \multicolumn{2}{|l|}{ Age } \\
\hline$<59$ years & $233(52.1)$ \\
\hline$\geq 59$ years & 214(47.9) \\
\hline \multicolumn{2}{|l|}{ Alcohol(n) } \\
\hline No & $261(58.4)$ \\
\hline Yes & 186(41.6) \\
\hline \multicolumn{2}{|l|}{ Stage(n) } \\
\hline I and II & $235(52.6)$ \\
\hline III and IV & $212(47.4)$ \\
\hline \multicolumn{2}{|l|}{ Treatment(n) } \\
\hline Surgery only & $238(53.3)$ \\
\hline Surgery and chemotherapy & 179(40.0) \\
\hline Surgery and radiotherapy & $6(1.3)$ \\
\hline $\begin{array}{l}\text { Surgery and chemotherapy and } \\
\text { radiotherapy }\end{array}$ & $24(5.4)$ \\
\hline \multicolumn{2}{|l|}{ Tumor location* } \\
\hline Upper & $40(9.0 \%)$ \\
\hline Middle & $278(62.1 \%)$ \\
\hline Lower & $128(28.6 \%)$ \\
\hline \multicolumn{2}{|l|}{$\operatorname{Dead}(\mathrm{n})$} \\
\hline No & 199(44.5) \\
\hline Yes & $248(55.5)$ \\
\hline \multicolumn{2}{|l|}{ Tests } \\
\hline $\mathrm{CRP}(\mathrm{mg} / \mathrm{L})^{\mathrm{a}}$ & $2.29(0.94-5.89)$ \\
\hline fibrinogen $(\mathrm{g} / \mathrm{L})^{\mathrm{b}}$ & $3.16(2.63-3.75)$ \\
\hline $\mathrm{TP}(\mathrm{g} / \mathrm{L})$ & $68.88(64.46-72.65)$ \\
\hline $\operatorname{ALB}(\mathrm{g} / \mathrm{L})$ & $42.75(40.28-44.80)$ \\
\hline $\mathrm{GLB}(\mathrm{g} / \mathrm{L})$ & $26.50(22.78-29.69)$ \\
\hline$A G{ }^{c}$ & $1.61(1.43-1.88)$ \\
\hline
\end{tabular}

${ }^{\mathrm{a}} \mathrm{n}=437,{ }^{\mathrm{b}} \mathrm{n}=428,{ }^{\mathrm{c}} \mathrm{n}=442$

*One patient has tumors both located in upper and lower esophagus.

\section{Results}

\section{Baseline characteristics in the study population and subgroups}

There were 447 patients pathologically confirmed with ESCC enrolled in this study (346 men and 101 women; ages $34-79$ years, median 59 years). In our cohort, all 447 patients underwent surgical resection. Specifically, 53.2\% $(238 / 447)$ patients underwent tumor resection without receiving any treatment, and $40.0 \%(179 / 447)$ patients received chemotherapy after surgery. Furthermore, $6(1.3 \%)$ patients received radiation and 24 (5.4\%) patients received radiation and chemotherapy after surgery. The characteristics and parameters of the patients are listed in Table 1.

\section{Prognostic significance of acute phase reactants in ESCC}

In the entire cohort, the median follow-up time was 52.0 (5.0 to 90.0) months and the median OS was 44.0 (1.0 to 87.0) months. At the end of observation, $55.5 \%(248 / 447)$ patients had died due to disease-related or unrelated factors.

To evaluate the prognostic value of acute phase proteins in ESCC, the demographic data, clinicopathological features, alcohol status and acute phase proteins were evaluated using univariate and multivariate Cox regression models (Table 2). The univariate analysis revealed that $\mathrm{T}$ classification, $\mathrm{N}$ classification, metastasis, TNM stage, alcohol history, treatment, the level of CRP, fibrinogen, ALB, and AGR were significantly associated with ESCC survival except for gender, age, TP and Glb. To eliminate the influence of collinearity, we excluded the factors of $\mathrm{T}$ classification, $\mathrm{N}$ classification, metastasis and Alb in the multivariate analysis. These remaining six variables were selected for multivariate analysis using a forward stepwise method. Consequently, the multivariate analysis showed that TNM stage (HR=3.050; 95\%CI: 2.323-4.005; $p<0.001)$, CRP (HR=1.447; 95\%CI: 1.039-2.014; $p=0.029$ ) and AGR (HR=1.320; 95\%CI: 1.008-1.728; $p=0.044$ ) were independent prognostic indicators of ESCC survival. Thus, the findings showed us that CRP and AGR before therapy may serve as a novel independent prognostic factor for ESCC.

To further explore the prognostic significance of AGR and CRP in ESCC, Kaplan-Meier survival curves were generated, and the groups were compared using the log-rank test. The patients were categorized into a low CRP group and a higher CRP group according to the cut-off value $10.0 \mathrm{mg} / \mathrm{L}$. Besides, the patients were also categorized into a low AGR group and a higher AGR group according to the cut-off value based on 
AGR level in the T1-T2 subgroup $(\mathrm{n}=123, p=0.016$, Fig. $3 \mathrm{~b}$ ) and T3-T4 subgroup ( $\mathrm{n}=319, p=0.041$, Fig. $3 c)$, N0 subgroup ( $n=207, p=0.000$, Fig. $3 d$ ), and M0 subgroup ( $n=427, p=0.002$, Fig. 3f).

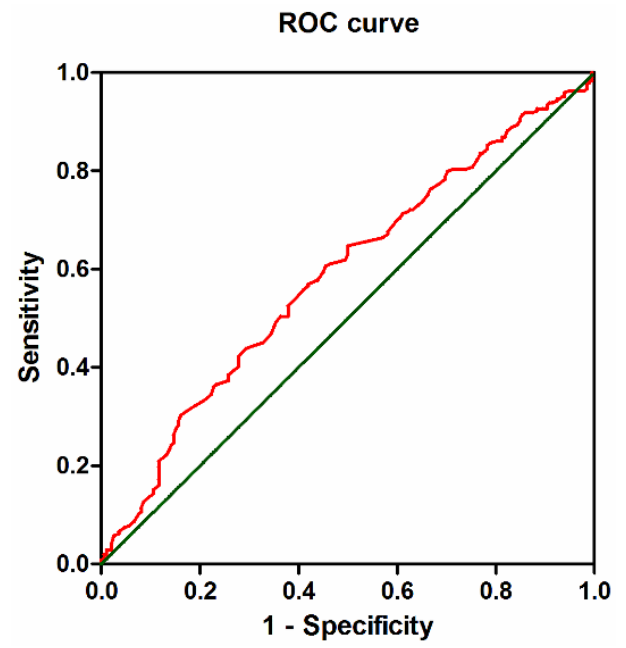

Figure 1. ROC curve of AGR. The Receiver operating characteristic curve analysis of the AGR in the patients with ESCC. The appropriate cutoff value for the AGR to be 1.66 (sensitivity: $54.5 \%$ and specificity: $60.7 \%$ ). Area under the curve $=0.597,95 \% \mathrm{Cl}: 0.534-0.640, p=0.002$.

\section{Relationship between AGR and clinical characteristics and acute phase proteins}

The associations between the AGR and clinical characteristics of patients are summarized in Table 3. The AGR was not associated with gender, age, alcohol history, histological differentiation, T classification, $\mathrm{N}$ classification or tumor stage except for CRP, fibrinogen and tumor metastasis. In the whole cohort, elevated AGR level was significant for the patients with normal CRP and fibrinogen level $(p<0.001$, respectively) and for those without tumor metastasis $(p=0.032)$. Furthermore, the patient cohort was divided into two groups: the higher AGR group and lower AGR group according to the cut-off value. Gender, age, alcohol history, histological differentiation, $\mathrm{T}$ classification, $\mathrm{N}$ classification and TNM stage were similar between the two groups. However, patients with a lower CRP and fibrinogen level were more common in the higher AGR group than in the lower AGR group. In other words, patients with a lower AGR tended to suffer inflammatory disease and hyperfibrinogenemia compared with the higher group ( $p<0.001$, respectively).

\section{Correlation of fibrinogen with AGR and their combining prognostic significance}

To confirm the relationship between the CRP, fibrinogen and AGR, we performed the Spearman's rank correlation analysis. The results revealed that the fibrinogen and AGR presented a negative correlation
( $\mathrm{r}=-0.317, p<0.001)$ (Fig. 4) while the CRP had no significant correlation with AGR.

To further explore the prognostic significance of combining fibrinogen and AGR, we generated Kaplan-Meier survival curves with the log-rank test for the whole cohort. The prognostic significance was shown in Fig. 5. The five-year overall survival rates of the four groups were significantly different $(p=$ 0.003). The patients with lower fibrinogen and higher AGR (group 3) had a significantly higher survival probability than the other three groups. Conversely, the survival rate of the patients with high fibrinogen and low AGR (group 2) was the lowest among the four groups. The sequence of the survival rates of the four groups from high to low was group 3(54.7\%)> group $1(39.2 \%)>$ group $4(39.1 \%)>$ group $2(33.9 \%)$.

\section{A nomogram predicts survival risk based on AGR and CRP}

In our study, we performed a nomogram model to predict the survival risk based on AGR, CRP and other prognostic factors. The nomogram model showed that the higher CRP and lower AGR made greater contribution to the lower survival rate than the other level (Fig. 6a). Based on our model, we believe that it could be a simple and easy tool for estimating the survival risk for patients with ESCC. In the current study, the Harrell's c-index was 0.687(0.654-0.72). Besides, the calibration curve for probability of 3-year OS and 5-year OS showed good agreement between prediction by nomogram and actual observation (Fig. 6b).

\section{Discussion}

Our study revealed that of the four acute phase reactants (CRP, ALB, fibrinogen and AGR), the pre-treatment AGR and CRP were demonstrated to be independent prognostic factors for overall survival in ESCC patients with tumor resection. We found that patients with a lower AGR or a higher CRP experienced significantly shorter overall survival than patients with a higher AGR or lower CRP, not only in the entire cohort but also in the subgroups stratified by $\mathrm{T}$ stage (T1-T2,T3-T4), $\mathrm{N}$ stage (N0), and metastasis (M0). Besides, the results were similar in CRP groups (group T3-T4,N0 and M0). The result of this particular study was consistent with previous studies that associated low serum AGR(or high GAR in reverse) with decreased long-term OS, not only in malignancies (including breast, lung, and colorectal cancer)[15-17, 19-21], but also in a generally healthy screened population for increasing cancer incidence and mortality[22]. All these evidences remind us that AGR is a potential index in predicting prognosis of tumor. However, researchers mainly focus on such 
inflammation indexes like C-reactive protein/ Albumin ratio, albumin-globulin score, fibrinogen, serum albumin levels or fibrinogen-albumin score (FA score) $[9,23,24]$ in esophageal cancer. In other
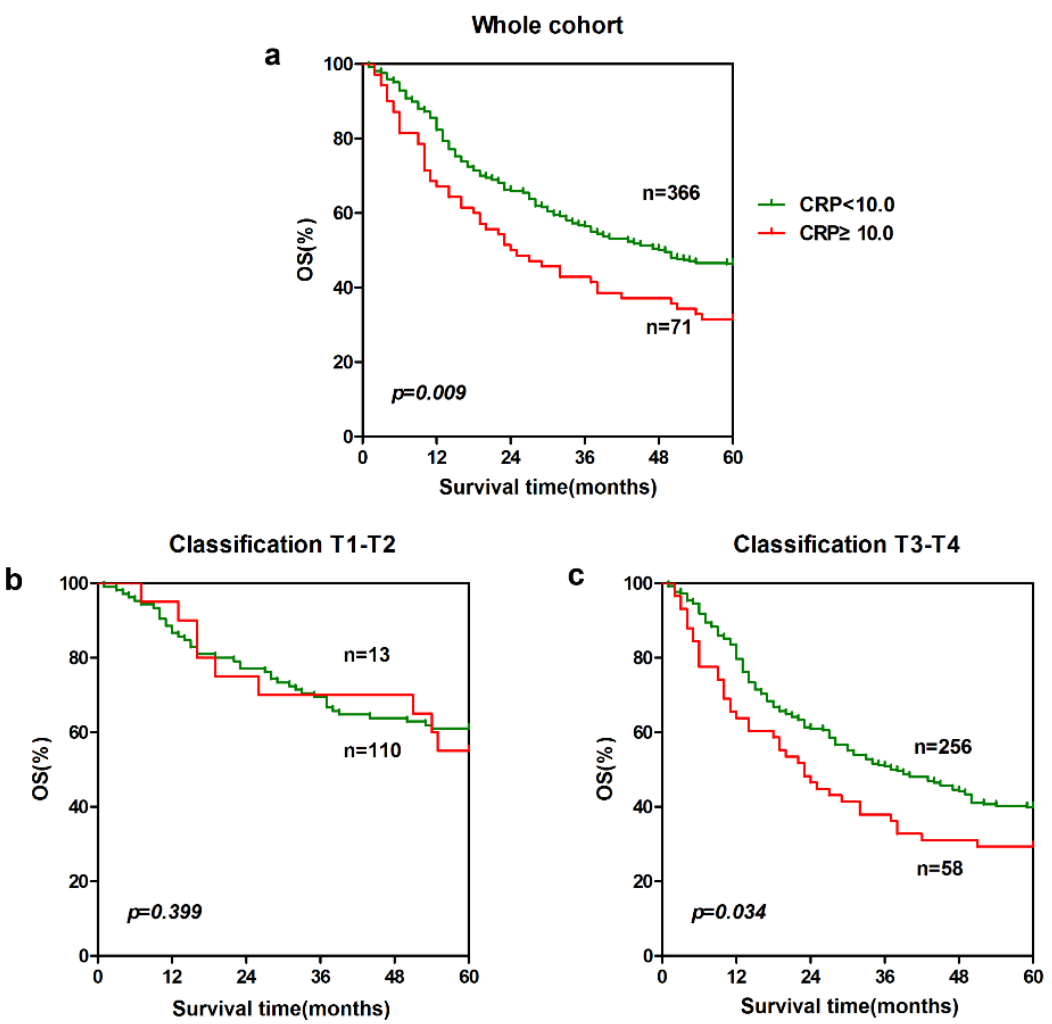

Classification NO
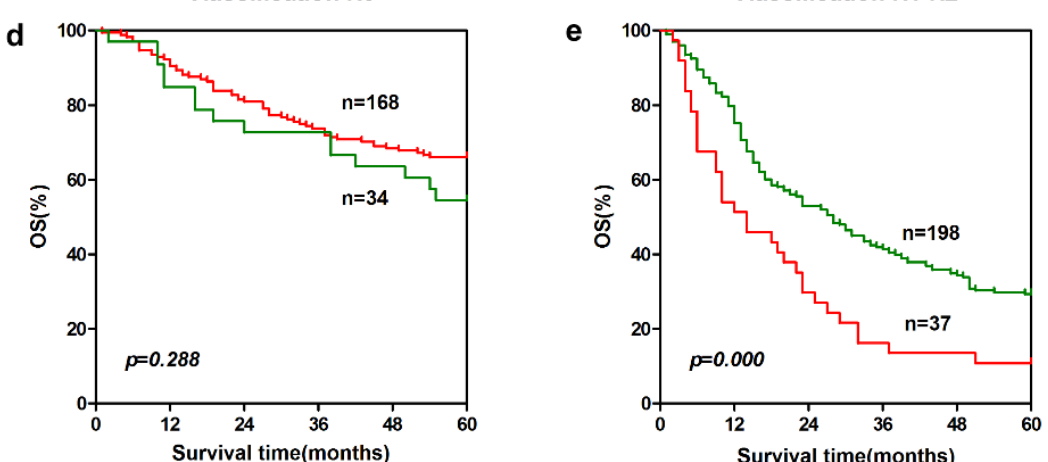

Classification MO

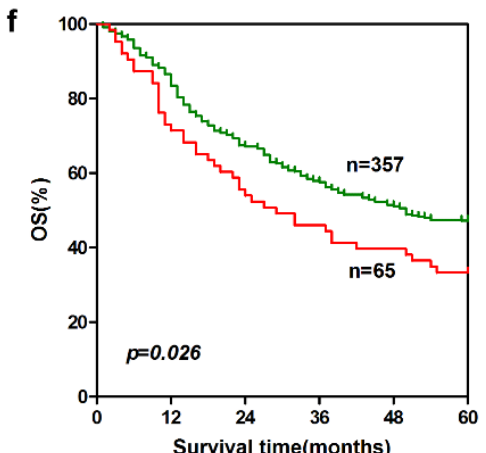

words, there are no clinical researches of the relationship between the AGR and patients with ESCC following surgical resection. To the best of our knowledge, this is the first study to assess the value of the AGR as a prognostic marker for ESCC and state the relationship between AGR and fibrinogen. Besides, differences were mainly found on T1-T2 subgroup, T3-T4 subgroup, N0 subgroup and M0 subgroup which suggested that the prognostic significance of AGR focus on patients without lymphatic or distant metastasis. For those with metastasis, AGR can not distinguish between the early stage tumor.

As major markers of systemic chronic inflammation and acute phase proteins, ALB is simple, inexpensive testes which is widely used in clinical laboratories. The production of albumin by hepatocytes is suppressed due to the activation of proinflammatory cytokines, including interleukin-1, interleukin-6 and tumor necrotic factor- $\mathrm{a}[25,26]$ when inflammation occurs. Globulin is a comprehensive protein which contains a kind of acute-phase proteins, such as C-reactive protein, serum amyloid A, complement C3, fibrinogen and ceruloplasmin[17]. As these proteins are produced in a state of inflammation, an increased level of globulin is thought to reflect the presence of continuous systemic inflammation. Some researcher found that globulin was suggested to be associated with the poor survival of hormone related cancer[27-29]. Both the serum albumin and serum globulin concentrations have been reported respectively to be prognostic index for survival in patients with various types of cancers[13, 14, 21]. However, in this present study, we chose AGR to evaluated the status of the host rather than individual parameter because AGR is more stable and our results were not interfered by the body status and several specific disease, such as dehydration, cachexy, and fluid retention[21].

Figure 2. Prognostic significance of CPR in ESCC. The five-year overall survival rate was calculated using the Kaplan-Meier method and analyzed with the log-rank test. A low CPR level was a favorable prognostic factor in the entire ESCC cohort. For CRP, the differences can be found in T3-T4 subgroup(c), N1-N2 subgroup (e) and M0 subgroup (f). 

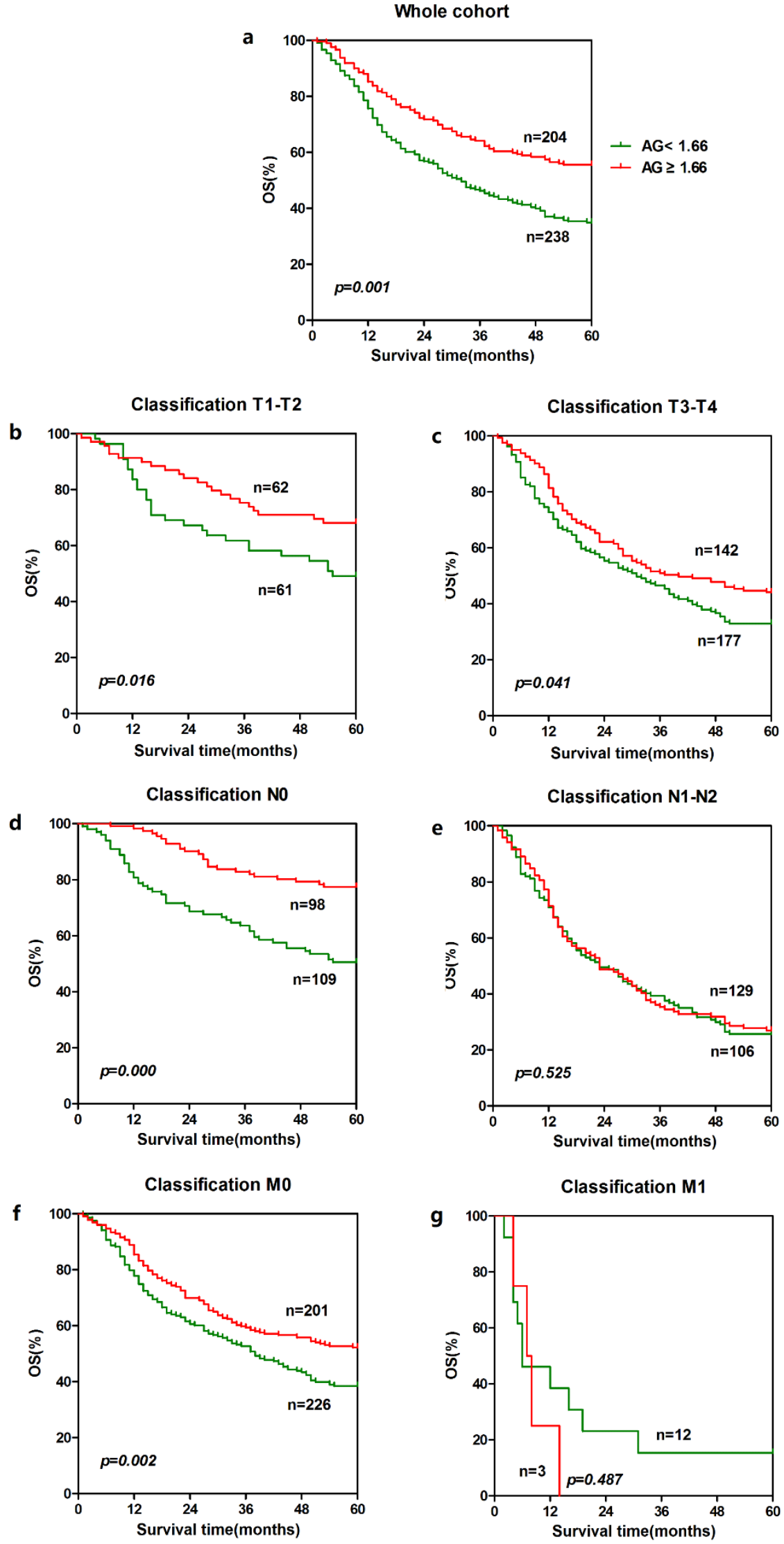

Figure 3. Prognostic significance of AGR in ESCC. The five-year overall survival rate was calculated using the Kaplan-Meier method and analyzed with the log-rank test. A high AGR level was a favorable prognostic factor in the entire ESCC cohort. For AGR, the differences can be found in T1-T2 subgroup(b), T3-T4 subgroup (c), N0 subgroup (d) and MO subgroup (f). 


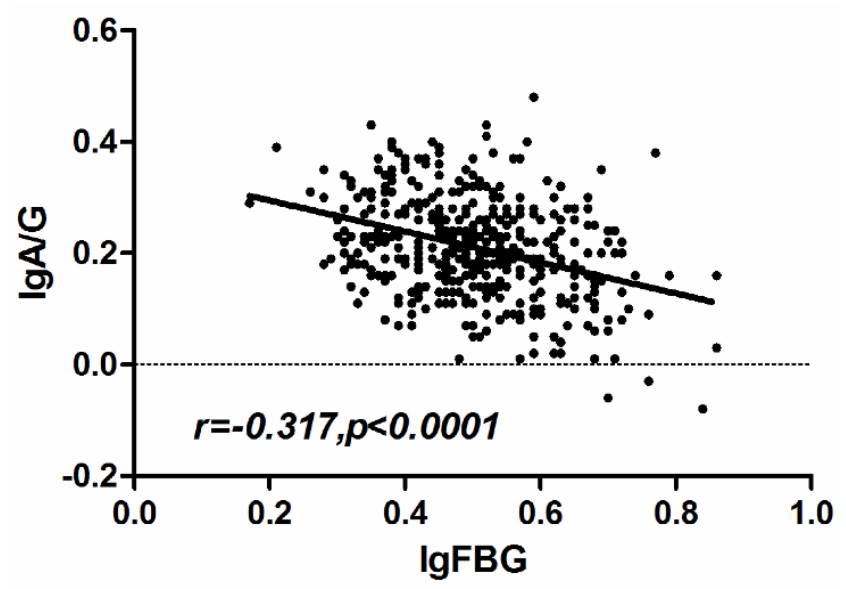

Figure 4. Correlation of fibrinogen and AGR in ESCC patients. The pre-therapy serum fibrinogen level was negatively correlated with AGR ( $r$ $=-3.17, p<0.001)$.

Moreover, more than $95 \%$ of patients have a normal albumin level in our study and patients with high ALB or GLB levels are uncommon. Fortunately, the AGR has been reported to be able to identify those expected to have a poor prognosis as we mentioned previously. Therefore, the AGR is considered to be a more accurate prognostic marker than the single serum albumin or globulin levels.

In addition to the prognostic value of AGR, we also revealed that the AGR and fibrinogen presented a negative correlation. The patients with lower fibrinogen and higher AGR had a significantly highest survival probability than the other three groups. The incidence of hyperfibrinogenemia in our study was $20.1 \%(86 / 428$, cut-off value $4.0 \mathrm{~g} / \mathrm{L})$. Researchers reported that hyperfibrinogenemia was positively correlated with tumor length, depth of invasion, pathological stage and disease recurrence in ESCC[30]. Morever, the elevated plasma fibrinogen level was identified as a predictive marker for decreased overall survival not only in the preoperative esophageal cancer[30,31], but also in the postoperative recurrence of ESCC patients who receiving neoadjuvant treatment[32]. Fibrinogen deposition around tumor cells enhances the interaction between these cells and platelets, which effectively form microemboli in target organs[33]. Fibrinogen layers help tumor cells block natural killer cytotoxicity with thrombin, which can protect tumor cells from the innate immune system[34]. In conclusion, hyperfibrinogenemia is negatively impact the course of the disease. It's interesting that in this present study, patients with lower fibrinogen and higher AGR had a significantly higher survival probability $(54.7 \%)$ than the other groups, especially than the higher fibrinogen and lower AGR group(33.9\%). Patients with a high preoperative fibrinogen and serum albumin levels (FA score) showed considerably shorter disease-free survival and overall survival in esophageal cancer[35] and we confirmed the existing conclusions coincidently in another way that serum fibrinogen level was negatively correlated with AGR.

The acute phase reaction is an element of non-specific immunity consisting of early reactions as a response to tissue damage, such as inflammation, infection, myocardial infarction and cancer. A special form of the acute phase reaction is the coordinated synthesis of several proteins, the so-called acute phase proteins. Acute phase proteins can be divided into positive and negative proteins, depending whether their concentration increases or decreases during the acute phase reaction. CRP, fibrinogen, GLB were considered as positive proteins while albumin are classified as negative proteins in our paper. Quantitative and qualitative acute phase protein changes observed during the neoplastic process remain unclear. They can result from the development of immunosuppression connected with the production of immunosuppressive cytokines by the tumor[36, 37]. In our study, patients with lower fibrinogen and higher AGR had a significantly higher survival probability than the higher fibrinogen and lower AGR group(Fig. 5b) which suggested that patients who had a slight inflammatory response when tumor occurred may had a better outcomes. In other words, patients had a good resistance to cancerinduced inflammation may had a ending. It's a pity that the specific mechanism why AGR and fibrinogen had a negative correlation is still unknown and it need to be further discussed in future study.

Unfortunately, fibrinogen wasn't an independent marker in resectable ESCC in our study although some researchers[38] found that fibrinogen was association with survival in operable esophageal cancer in which patients with hyperfibrinogenemia exhibited poor OS and DFS. CRP was found to be an independent prognostic indicator in out study and it is widely recognized for numerous cancers[39-43], including esophageal cancer[44-47]. Out result is similar than that reported in other ESCC studies.

\section{Conclusions}

In this study, we demonstrated that the AGR is associated with the overall survival in ESCC with surgery. Patients with a low AGR are considered to be a risk factor in tumor ending. In addition, the pre-therapy serum fibrinogen level was negatively correlated with AGR which indicates that the combination of fibrinogen and AGR maybe a high-efficiency index in prognosis of cancer. 

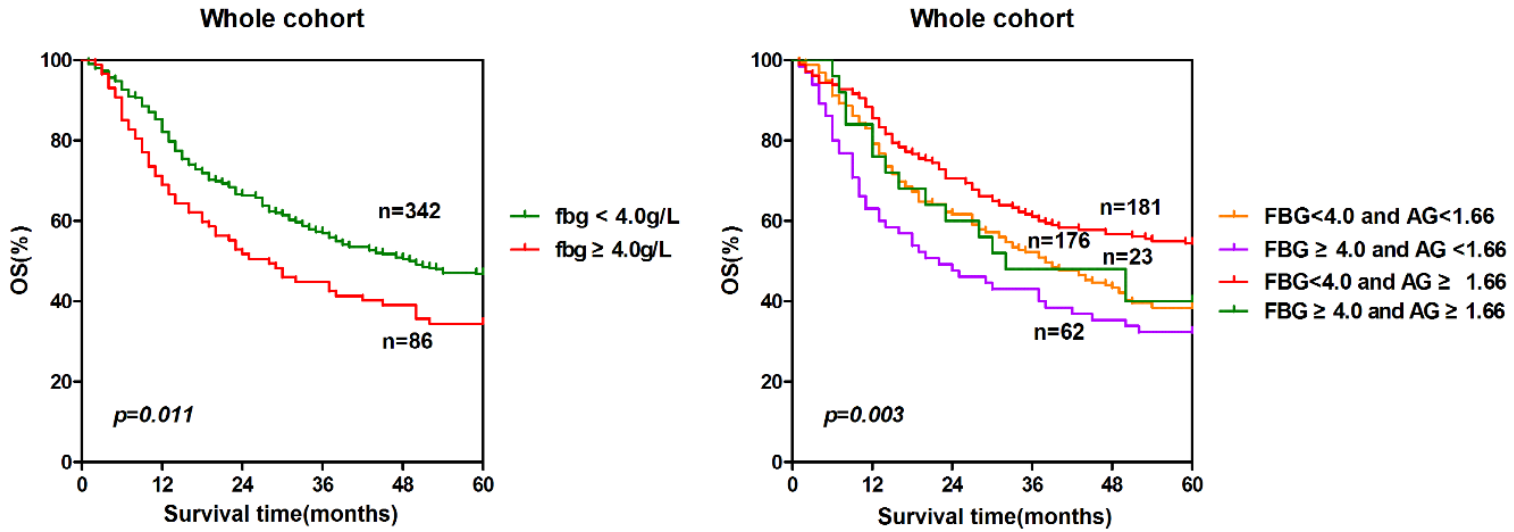

Figure 5. Prognosis significance of combining fibrinogen and AGR. The patients were classified to four groups according to the cut-off values of fibrinogen $(4.0 \mathrm{~g} / \mathrm{L})$ and AGR (1.66). Group 1: low fibrinogen and low AGR; group 2: high fibrinogen and low AGR; group 3: low fibrinogen and high AGR; group 4: high fibrinogen and high AGR. The five-years overall survival rates of the four groups were calculated by the Kaplan-Meier method and analyzed by the log-rank test.

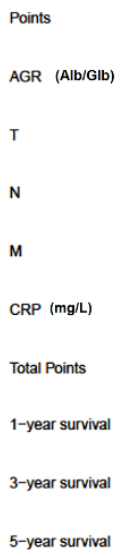

b

\section{A nomogram predicts survival risk based on AGR and CRP}

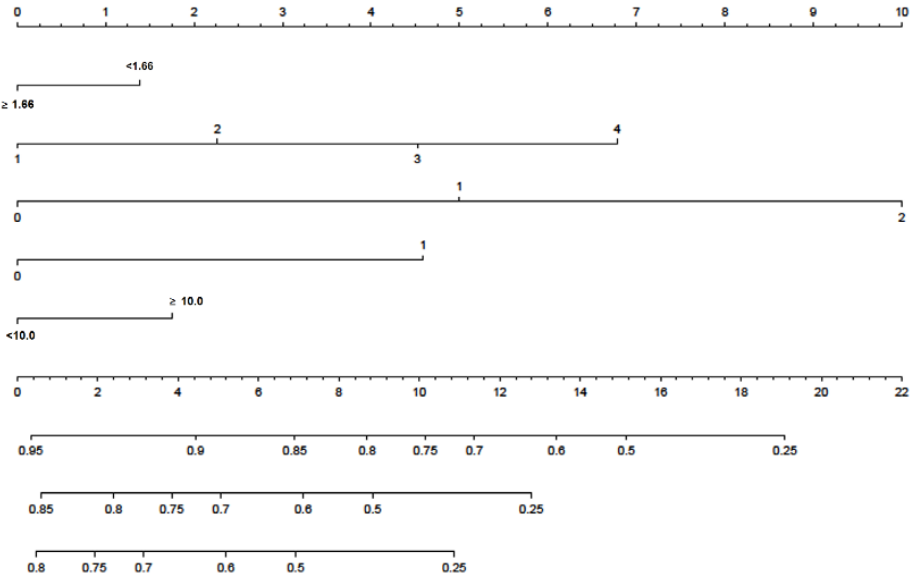

Calibration graphs for the 3-year OS and 5-year OS
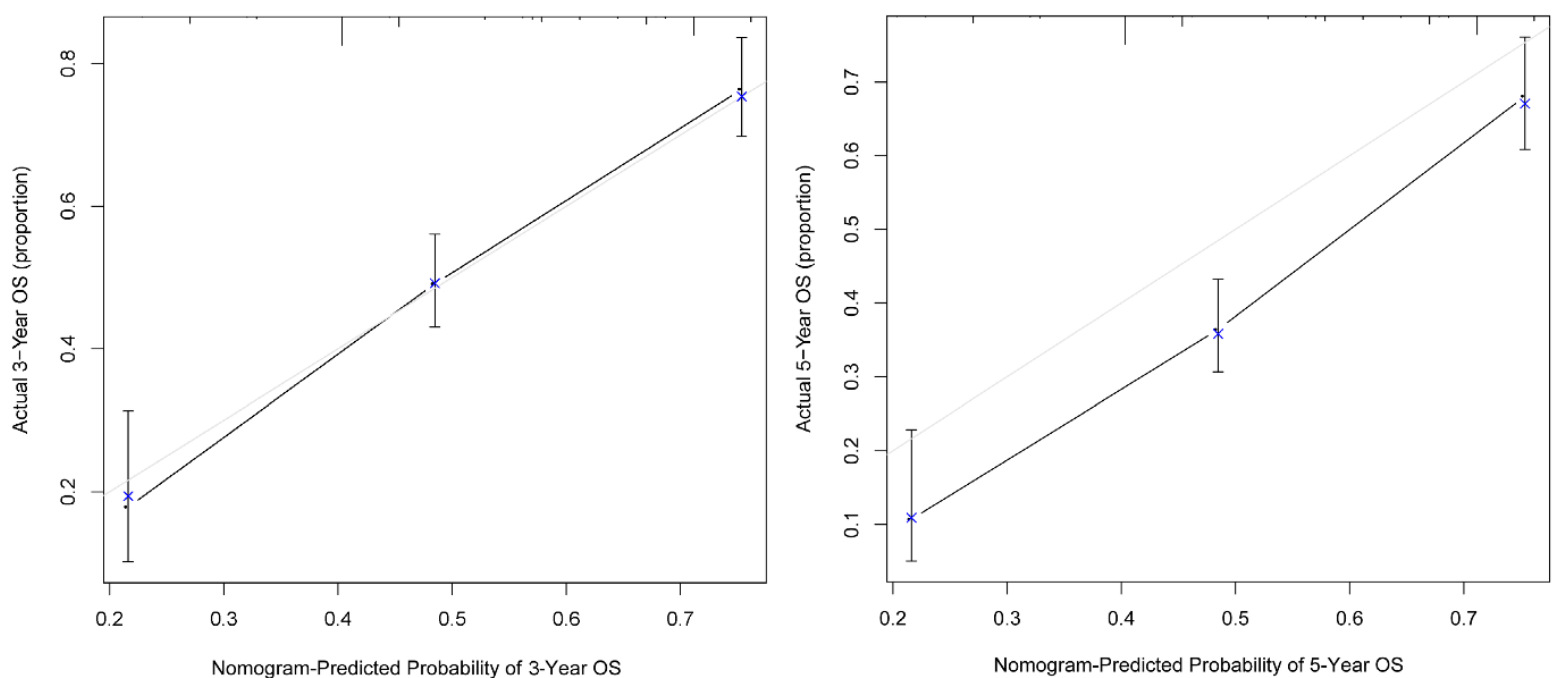

Figure 6. A nomogram predicts survival risk based on AGR and CRP(a) and Calibration graphs for the 3-year OS and 5-year OS(b). The nomogram is used by totaling the points identified at the top of the scale for each independent factor. This total point score is then identified on the total points scale to determine the probability of risk prediction. The Harrell's c-index for OS prediction was 0.687 (a). The calibration curve for probability of 3-year OS and 5-year OS showed good agreement between prediction by nomogram and actual observation(b). 


\section{Acknowledgment}

We thank the staff at the Director of Clinical Laboratories, Sun Yat-sen University Cancer Center, for providing support on research conditions in this study. This work was partly funded by the Guangdong Esophageal Cancer Research Institute [grant number: Q201405].

\section{Competing Interests}

All authors declare no conflicts of interest. Informed consent was obtained from all individual participants included in the study.

\section{References}

[1]. Lin Y., et al. Epidemiology of esophageal cancer in Japan and China. J Epidemiol, 2013. 23(4): 233-42.

[2]. Arnold M., et al. Global incidence of oesophageal cancer by histological subtype in 2012. Gut, 2015. 64(3): 381-7.

[3]. Ke L. Mortality and incidence trends from esophagus cancer in selected geographic areas of China circa 1970-90. Int J Cancer, 2002. 102(3): 271-4.

[4]. McMillan, D.C., The systemic inflammation-based Glasgow Prognostic Score: a decade of experience in patients with cancer. Cancer Treat Rev, 2013. 39(5): $534-40$

[5]. Sun K., et al. The prognostic significance of the prognostic nutritional index in cancer: a systematic review and meta-analysis. J Cancer Res Clin Oncol, 2014. 140(9): 1537-49.

[6]. Paramanathan A., et al. A systematic review and meta-analysis on the impact of pre-operative neutrophil lymphocyte ratio on long term outcomes after curative intent resection of solid tumours. Surg Oncol, 2014. 23(1): 31-9.

[7]. Dutta S., et al. Comparison of the prognostic value of tumour- and patient-related factors in patients undergoing potentially curative resection of oesophageal cancer. World J Surg, 2011. 35(8): 1861-6.

[8]. Feng J.F, and Chen Q.X. Significance of the prognostic nutritional index in patients with esophageal squamous cell carcinoma. Ther Clin Risk Manag, 2014. 10: 1-7.

[9]. Wei X.L., et al. A novel inflammation-based prognostic score in esophageal squamous cell carcinoma: the C-reactive protein/albumin ratio. BMC Cancer, 2015. 15: 350.

[10]. Xu X.L., et al. A Novel Inflammation-Based Prognostic Score, the C-Reactive Protein/Albumin Ratio Predicts the Prognosis of Patients with Operable Esophageal Squamous Cell Carcinoma. PLoS One, 2015. 10(9): e0138657.

[11]. Kinoshita A., et al. The C-reactive protein/albumin ratio, a novel inflammation-based prognostic score, predicts outcomes in patients with hepatocellular carcinoma. Ann Surg Oncol, 2015. 22(3): 803-10.

[12]. Kasprzyk M., et al. [The assessment of acute phase proteins as prognostic factors in patients surgically treated for non-small cell lung cancer]. Pneumonol Alergol Pol, 2008. 76(5): 321-6.

[13]. Onate-Ocana L.F., et al. Serum albumin as a significant prognostic factor for patients with gastric carcinoma. Ann Surg Oncol, 2007. 14(2): 381-9.

[14]. Boonpipattanapong $\mathrm{T}$ and Chewatanakornkul S. Preoperative carcinoembryonic antigen and albumin in predicting survival in patients with colon and rectal carcinomas. J Clin Gastroenterol, 2006. 40(7): 592-5.

[15]. Zhou T., et al. Pretreatment Albumin/Globulin Ratio Predicts the Prognosis for Small-Cell Lung Cancer. Medicine (Baltimore), 2016. 95(12): e3097.

[16]. Shibutani, M., et al. The pretreatment albumin to globulin ratio predicts chemotherapeutic outcomes in patients with unresectable metastatic colorectal cancer. BMC Cancer, 2015. 15: 347.

[17]. Azab B., et al. The value of the pretreatment albumin/globulin ratio in predicting the long-term survival in colorectal cancer. Int J Colorectal Dis, 2013. 28(12): 1629-36.

[18]. Rice T.W., et al, 7th edition of the AJCC Cancer Staging Manual: esophagus and esophagogastric junction. Ann Surg Oncol, 2010. 17(7): 1721-4.

[19]. Park S., et al. Pretreatment albumin-to-globulin ratio as a predictive marker for tyrosine kinase inhibitor in non-small cell lung cancer. Cancer Biomark, 2016. 16(3): 425-33.

[20]. Yao Y., et al. Elevated pretreatment serum globulin albumin ratio predicts poor prognosis for advanced non-small cell lung cancer patients. J Thorac Dis, 2014. 6(9): 1261-70

[21]. Azab B.N., et al. Value of the pretreatment albumin to globulin ratio in predicting long-term mortality in breast cancer patients. Am J Surg, 2013. 206(5): 764-70.

[22]. Suh B., et al. Low albumin-to-globulin ratio associated with cancer incidence and mortality in generally healthy adults. Ann Oncol, 2014. 25(11): 2260-6.
[23]. Zhang F., et al. Low preoperative albumin-globulin score predicts favorable survival in esophageal squamous cell carcinoma. Oncotarget, 2016. 7(21): 30550-60.

[24]. Matsuda S., et al. Cumulative prognostic scores based on plasma fibrinogen and serum albumin levels in esophageal cancer patients treated with transthoracic esophagectomy: comparison with the Glasgow prognostic score. Ann Surg Oncol, 2015. 22(1): 302-10.

[25]. McMillan D.C., et al. Albumin concentrations are primarily determined by the body cell mass and the systemic inflammatory response in cancer patients with weight loss. Nutr Cancer, 2001. 39(2): 210-3.

[26]. Chojkier M.. Inhibition of albumin synthesis in chronic diseases: molecular mechanisms. J Clin Gastroenterol, 2005. 39(4 Suppl 2): S143-6.

[27]. Kristal A.R., et al. Serum steroid and sex hormone-binding globulin concentrations and the risk of incident benign prostatic hyperplasia: results from the prostate cancer prevention trial. Am J Epidemiol, 2008. 168(12): 1416-24.

[28]. Naik S.L.D., et al. Sex hormone binding globulin in breast cancer. Indian J Clin Biochem, 2008. 23(3): 250-4.

[29]. Thompson D.J., et al. Identification of common variants in the SHBG gene affecting sex hormone-binding globulin levels and breast cancer risk in postmenopausal women. Cancer Epidemiol Biomarkers Prev, 2008. 17(12): 3490-8.

[30]. Wang J., et al. The clinical significance of preoperative plasma fibrinogen level and platelet count in resectable esophageal squamous cell carcinoma. World J Surg Oncol, 2015. 13: 157.

[31]. Zhang S.S., et al. Preoperative serum fibrinogen is an independent prognostic factor in operable esophageal cancer. Oncotarget, 2016. 7(18): 25461-9.

[32]. Ilhan-Mutlu, A., et al. Plasma fibrinogen and blood platelet counts are associated with response to neoadjuvant therapy in esophageal cancer. Biomark Med, 2015. 9(4): 327-35.

[33]. Shimada H., et al. Thrombocytosis associated with poor prognosis in patients with esophageal carcinoma. J Am Coll Surg, 2004. 198(5): 737-41.

[34]. Zheng S., et al. Platelets and fibrinogen facilitate each other in protecting tumor cells from natural killer cytotoxicity. Cancer Sci, 2009. 100(5): 859-65.

[35]. Matsuda S., et al. Cumulative prognostic scores based on plasma fibrinogen and serum albumin levels in esophageal cancer patients treated with transthoracic esophagectomy: comparison with the Glasgow prognostic score. Ann Surg Oncol, 2015. 22(1): 302-10.

[36]. Smith D.R., et al. Production of interleukin-10 by human bronchogenic carcinoma. Am J Pathol, 1994. 145(1): 18-25.

[37]. Atwell, D.M., et al. Balance of proinflammatory and antiinflammatory cytokines at thoracic cancer operation. Ann Thorac Surg, 1998. 66(4): 1145-50.

[38]. Zhang S.S., et al. Preoperative serum fibrinogen is an independent prognostic factor in operable esophageal cancer. Oncotarget, 2016.

[39]. Szturmowicz M., et al. Prognostic value of serum C-reactive protein (CRP) and cytokeratin 19 fragments (Cyfra 21-1) but not carcinoembryonic antigen (CEA) in surgically treated patients with non-small cell lung cancer. Pneumonol Alergol Pol, 2014. 82(5): 422-9.

[40]. Dobrzycka B., et al. Serum levels of IL-6, IL-8 and CRP as prognostic factors in epithelial ovarian cancer. Eur Cytokine Netw, 2013. 24(3): 106-13.

[41]. Steffens S., et al. High CRP values predict poor survival in patients with penile cancer. BMC Cancer, 2013. 13: 223.

[42]. Juan H., et al. Serum CRP protein as a differential marker in cancer. Cell Biochem Biophys, 2012. 64(2): 89-93.

[43]. Onitilo A.A., et al. High-sensitivity C-reactive protein (hs-CRP) as a biomarker for trastuzumab-induced cardiotoxicity in HER2-positive early-stage breast cancer: a pilot study. Breast Cancer Res Treat, 2012. 134(1): 291-8.

[44]. Verma G.R., et al. Thrombocytosis and Raised CRP Levels Predicts Advanced Stage in Esophageal Carcinoma. J Gastrointest Cancer, 2015. 46(4): 350-5.

[45]. Nakatsu T., et al. Tumoral CRP expression in thoracic esophageal squamous cell cancers is associated with poor outcomes. Surg Today, 2012. 42(7): 652-8.

[46]. Lukaszewicz-Zajac M., et al. Comparative evaluation of serum C-reactive protein (CRP) levels in the different histological subtypes of esophageal cancer (squamous cell carcinoma and adenocarcinoma of esophagus). J Clin Lab Anal, 2012. 26(2): 73-81.

[47]. Fujiwara H., et al. Elevated serum CRP levels after induction chemoradiotherapy reflect poor treatment response in association with IL-6 in serum and local tumor site in patients with advanced esophageal cancer. J Surg Oncol, 2011. 103(1): 62-8. 